\title{
Biosensors Using an Antibody as a Recognition Element
}

\author{
Tomoyuki YASUKAWA
}

\author{
Graduate School of Material Science, University of Hyogo, 3-2-1 Kouto, Kamigori, Ako, Hyogo 678-1297, Japan
}

An antibody has frequently been used as a recognition element for developing biosensors due to its large variety, low dissociation constant and high specificity. However, there is no ability to covert the recognition events to the signal directly. Thus, a wide variety of signal conversion mechanisms have been studied for developing simple, rapid, and sensitive sensing systems in the medical, environmental and food analytical fields. ${ }^{1,2}$ The microfluidic systems used to separate unbounded molecules automatically and the difference of the signal index before and after the recognition events, e.g. the aggregation of nanoparticles by trapping target molecules, ${ }^{3}$ are applied for both simplicity and rapidity. Regarding sensitivity, production systems involving a large amount of signal molecules per single recognition event are significantly effective. Here, recent developments are introduced in the present highlight.

Fuchiwaki et al. described a novel lateral-flow test chip that can perform operations similar to a pump, such as flowing, stopping, and replacing a solution, just by adding the solution onto an inlet port. ${ }^{4}$ A transparent film was applied to the detection area of the chip so as to detect weak light generated by a specific antigen-antibody reaction. The limit of detection achieved by the present chip was three orders of magnitude lower than that by a nitrocellulose membrane. Tominaga et al. described a single-step electrochemical immunochromatography used enzyme reaction of glucose oxidase (GOx) as a label. ${ }^{5}$ The reaction of uncaptured GOx was successfully detected after labeled GOx was separated by the antibody immobilized on the membrane. Hirayama et al. reported an immunosensor based on surface plasmon resonance to detect three pesticide residues (boscalid, clothianidin and nitenpyram) in vegetables simultaneously. ${ }^{6}$ They designed three channels modified with different pesticides, and evaluated the availability of the developed sensor. Yin et al. reported on aptamer-based colorimetric sensors for Ochratoxin A. ${ }^{7}$ The adsorption of Ochratoxin A to gold nanoparticles protect the aggregation of particles in the absence of a target, while the aggregation was caused by specific recognition of aptamers in the presence of a target. Shan et al. described determining the binding constant of the complementary cavity for a whole cell. ${ }^{8}$ They introduce
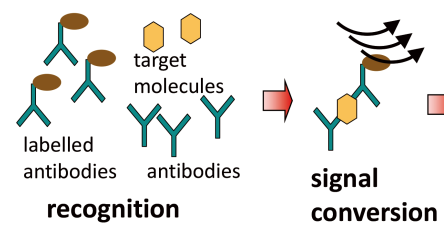

$\checkmark$ Rapidity \& Simplicity

-Automatic separation of unbounded molecules to antibody

- Difference of the signal index before and after the recognition events

$\checkmark$ Sensitivity

- Production of large amount of signa molecules per single recognition event

Signal conversion following a recognition event of an antibody.

E-mail: yasu@ sci.u-hyogo.ac.j a procedure to determine the binding constant of a cell-shaped cavity formed on a polymer by molecular imprinting for a whole cell. Lv et al. reported the determination of an aryl hydrocarbon receptor (AhR) coupled with anthracene (Ant) that exhibits genotoxicity and carcinogenicity based on an indirect competition enzyme linked immunosorbent assay using an AhR-Ant antibody. ${ }^{9}$ The detection range $\left(10-1000 \mathrm{ng} \mathrm{mL}^{-1}\right)$, the limit of detection $\left(2.43 \mathrm{ng} \mathrm{mL}^{-1}\right)$, the cross reaction ratio $(0.1-19.1 \%)$ and the recovery range (90 to $115 \%)$ were reported.

Song et al. described a highly sensitive analysis method based on silver metal deposition on gold particles conjugated with antibodies that was immobilized on graphite-like carbon nitride. $^{10}$ The detection limit is as low as $10^{2} \mathrm{CFU} \mathrm{mL}{ }^{-1}$, exhibiting an increase of two orders of magnitude compared with the traditional amplification by silver deposition. Batistela et al. also described a simple colorimetric immunoassay based on the aggregation inhibition of silver nanoparticles functionalized with an antibody on the surface. ${ }^{11}$ A bilayer lipid membrane sensor for bovine serum albumin has been developed based on a membrane-bound receptor approach using gramicidin channels by Zamoto et al. ${ }^{12}$ A successful determination with a detection limit of $\mathrm{pg} \mathrm{mL}^{-1}$ level was described. Biorecognition by an antibody has been applied to detect a prostate-specific antigen (PSA) based on a sol-gel integrated affinity microarray for on-chip matrix-assisted laser desorption/ionization time-offlight mass spectrometry (MALDI-TOF-MS). ${ }^{13}$ Megger et al. described the application of tandem mass tags (TMT) for the labeling of whole intact cells. ${ }^{14}$ The enrichment of TMT protein by an anti-TMT antibody was applied to increase the relative amount of labeled protein at the levels of whole cells. The development of a mass spectrometry-based bioanalytical method for therapeutic monoclonal antibodies and antibody-drug conjugates has also been reviewed. ${ }^{15}$

The development of novel signal conversion systems utilizing an antibody with excellent properties is of great interest because it permits the simple, rapid, and sensitive sensing of proteins related to diseases, environmental pollutants and pathogens.

Keywords Antibody, recognition event, signal conversion

\section{References}

1. B. Byrne, E. Stack, N. Gilmartin, and R. O'Kennedy, Sensors, 2009, 9, 4407.

2. V. Perumal and U. Hashim, J. Appl. Biomed., 2014, 12, 1.

3. K. Omidfar, F. Khorsand, M. Darziani Azizi, Biosens. Bioelectron., 2013, 43, 336.

4. Y. Fichiwali, K. Goya, and M. Tanaka, Anal. Sci., 2018, 34, 57.

5. K. Tominaga, S. Arimoto, K. Shimono, T. Yoshioka, F. Mizutani, and T. Yasukawa, Anal. Sci., 2017, 33, 531. 
6. Y. Hirakawa, T. Yamasaki, A. Harada, S. Iwasa, H. Narita, and S. Miyake, Anal. Sci., 2018, 34, 533.

7. X. Yin, S. Wang, X. Liu, C. He, Y. Tang, Q. Li, J. Liu, H. Su, T. Tan, and Y. Dong, Anal. Sci., 2017, 33, 659.

8. X. Shan, T. Yamauchi, H. Shiigi, and T. Nagaoka, Anal. Sci., 2018, 34, 483.

9. Y. Lv, X. Zhao, L. Yang, X. Zhang, and Y. Bai, Anal. Sci., 2018, 34, 421.

10. X. Song, Y. Wu, L. Wu, Y. Hu, W. Li, Z. Guo, X. Su, and X. Jiang, Anal. Sci., 2017, 33, 889.
11. D. M. Batistela, C. V. Stevani, and R. S. Freire, Anal. Sci. 2017, 33, 1111.

12. T. Zamoto, S. Tominaga, M. Nishio, A. Shoji, and M. Sugawara, Anal. Sci., 2017, 33, 1421.

13. S.-W. Lee, J.-H. Lee, H.-G. Kwon, T. Laurell, O.-C. Jeong, and S. Kim, Anal. Sci., 2018, 34, 317.

14. D. A. Megger, L. L. Pott, K. Rosowski, B. Zülch, S. Tautges, T. Bracht, and B. Sitek, Anal. Sci., 2017, 33, 1387.

15. K. Todoroki, T. Yamada, H. Mizuno, and T. Toyo' oka, Anal. Sci., 2018, 34, 397. 